\title{
The decoupled nature of basal metabolic rate and body temperature in endotherm evolution
}

Article

Accepted Version

Avaria-Llautureo, J., Hernández, C. E., Rodríguez-Serrano, E. and Venditti, C. (2019) The decoupled nature of basal metabolic rate and body temperature in endotherm evolution. Nature, 572. pp. 651-654. ISSN 0028-0836 doi:

https://doi.org/10.1038/s41586-019-1476-9 Available at https://centaur.reading.ac.uk/85675/

It is advisable to refer to the publisher's version if you intend to cite from the work. See Guidance on citing.

To link to this article DOI: http://dx.doi.org/10.1038/s41586-019-1476-9

Publisher: Nature Research

All outputs in CentAUR are protected by Intellectual Property Rights law, including copyright law. Copyright and IPR is retained by the creators or other copyright holders. Terms and conditions for use of this material are defined in the End User Agreement.

www.reading.ac.uk/centaur 
Central Archive at the University of Reading

Reading's research outputs online 
1 Title: The decoupled nature of basal metabolic rate and body temperature in endotherms

2 evolution

4 Authors: Jorge Avaria-Llautureo ${ }^{1,4}$, Cristián E. Hernández ${ }^{2}$, Enrique Rodríguez-Serrano ${ }^{3}$, 5 Chris Venditti ${ }^{1}$.

6

7 Author affiliations:

8

9 1. School of Biological Sciences, University of Reading, Reading, RG6 6BX, United 10 Kingdom.

11

12 2. Laboratorio de Ecología Evolutiva y Filoinformática, Departamento de Zoología,

13 Facultad de Ciencias Naturales y Oceanográficas, Universidad de Concepción,

14 Concepción, Chile.

15

16 3. Laboratorio de Mastozoología, Departamento de Zoología, Facultad de Ciencias

17 Naturales y Oceanográficas, Universidad de Concepción, Concepción, Chile.

18

19 4. Facultad de Ciencias. Universidad Católica de la Santísima Concepción (UCSC).

20 Centro de Investigación en Biodiversidad y Ambientes Sustentables (CIBAS). Chile. 
The origin of endothermy in birds and mammals are iconic events in vertebrate evolution. Endotherms can maintain their body temperature $\left(T_{b}\right)$ over a wide range of ambient temperatures $\left(T_{\text {a }}\right)$ using primarily the heat generated continuously by their high basal metabolic rates $(B M R)^{1}$. There is also an important positive feedback loop in that $B M R$ itself is influenced by $T_{b^{1-3}}$. Owing to this, many ecologists and evolutionary physiologists posit that the evolution of $B M R$ and $T_{b}$ must have been coupled during the radiation of endotherms ${ }^{3-5}$, changing with similar trends ${ }^{6-8}$. However, colder historical environments might have imposed strong selective pressures on $B M R$ to compensate for increased rates of heat loss and to keep $T_{\mathrm{b}}$ constant ${ }^{9-12}$. Thus, adaptation to cold $T_{\mathrm{a}}$ via $B M R$ increases could have decoupled $B M R$ from $T_{\mathrm{b}}$ and caused different evolutionary routes to the modern diversity in these traits. Here we show that $B M R$ and $T_{b}$ were decoupled in $\sim 90 \%$ of mammalian and in $\sim 36 \%$ of avian phylogenetic branches. Mammalian $B M R$ evolved with rapid bursts but without any long-term directional trend, whereas $\boldsymbol{T}_{\mathrm{b}}$ evolved mostly at a constant rate and towards colder bodies from a warmer-bodied ancestor. Avian $B M R$ evolved predominantly at a constant rate - again with no trend, whereas $T_{b}$ evolved with much greater rate heterogeneity than $B M R$ and there has been adaptive evolution towards colder bodies. Furthermore, rapid shifts leading to both $B M R$ increases and decreases were linked to abrupt changes towards colder $\boldsymbol{T}_{\mathrm{a}}$ but only in mammals. Our results suggest that natural selection effectively exploited the diversity of mammalian BMR under diverse, often adverse historical thermal environments.

Phylogenetic statistical methods ${ }^{13,14}$ now provide us with the opportunity to formally test whether $B M R$ has been linked to $T_{\mathrm{b}}$ or $T_{\mathrm{a}}$ throughout the evolution of birds and mammals. By accommodating for and identifying heterogeneity in the rate of phenotypic evolution

48 these methods can detect and reconstruct accurate historical evolutionary processes ${ }^{15}$.

49 Evaluation of the evolutionary coupling between $B M R$ and $T_{\mathrm{b}}$ has direct consequences for

50 several longstanding ecological and evolutionary theories (including the Metabolic

51 Theory of Ecology) which assume coupling ${ }^{2-8}$.

53 We first quantified and compared rates for $B M R$ and $T_{\mathrm{b}}$ evolution along each branch of

54 the time-calibrated phylogenetic trees of birds and mammals (henceforth branch-wise

55 rates, $r$; see Methods). $r$ measures how fast a trait evolved along an individual 
56 phylogenetic branch ( $r$ is a rate scalar by which the background rate is multiplied to

57 increase or decrease the pace of evolution). If $B M R$ and $T_{\mathrm{b}}$ were coupled during the

58 evolution of endotherms, the amount of change along phylogenetic branches in both traits

59 should be positively associated - where $r$ is high in $B M R$ we expect it to be high in $T_{\mathrm{b}}$

60 (Fig. 1 b). We tested this prediction against alternative evolutionary scenarios. Firstly, we

61 cannot make any inferences about coupling or decoupling where there is no rate

62 heterogeneity for both $B M R$ and $T_{\mathrm{b}}(r=1$ for all branches in the tree for both traits; Fig.

63 1a). Secondly, we infer decoupled evolution if both traits show rate heterogeneity, but the

64 magnitudes of $r$ s are negatively correlated (i.e. branches evolving at a high rate for $B M R$

65 are evolving at a low rate for $T_{\mathrm{b}}$, and vice-versa, Fig. 1c). We suggest this scenario

66 implies decoupled evolution because a negative correlation most likely implies that one

67 trait tends to be conserved whilst the other evolved rapidly. Thirdly, we infer decoupled

68 evolution if only one trait shows rate heterogeneity while the other evolved at a constant

69 rate (Fig $1 \mathrm{~d}$ and e) or if both traits show heterogeneity but the branch-wise rates are not

70 associated (Fig 1f).

72 As BMR, body mass (Mass), $T_{\mathrm{b}}$, and $T_{\mathrm{a}}$ are at least to some extent correlated in extant

73 birds and mammals, and such correlations may vary between orders ${ }^{16}$, we estimated the

74 branch-wise rates for $B M R$ and $T_{\mathrm{b}}$ while accounting for their covariates across extant

75 species using the phylogenetic variable-rates regression mode $1^{17}$ (henceforth variable-

76 rates; Methods). This approach allows for simultaneous estimation of both an overall

77 relationship between, for instance, $B M R$ as a function of Mass and $T_{\mathrm{b}}$ across extant

78 species, and any shifts in rates ( $r$ ) that apply to the phylogenetically structured residual 
79 variance in the relationship. In both birds and mammals, the variable-rates model

80 significantly fits the data better than the constant-rate regressions, which assume a single

81 rate across all branches (Methods; Table S1 to S8). The best fitting variable-rates model

82 for mammalian BMR includes both Mass and $T_{\mathrm{b}}$ with a single slope for each trait

83 estimated across all orders (Table S1 and S2). For mammalian $T_{\mathrm{b}}$, the best fitted model

84 includes Mass and BMR as covariates, also with a single slope across all orders (Table S3

85 and S7). In birds, the best model for BMR includes only Mass with a single slope for all

86 orders (Table S4). Finally, the best fitted model for avian $T_{\mathrm{b}}$ includes Mass only in

87 Columbiformes (Table S6).

89 The branch-wise rates estimated in the best fitting models shows that mammalian BMR

90 evolved at a constant rate $(r=1)$ in just $11.2 \%$ of branches and at faster rates $(r>1)$ in

$9188.8 \%$ of branches (Fig. 2a). Mammalian $T_{\mathrm{b}}$ evolved at a constant rate in $70.3 \%$ of

92 branches and faster rates in $29.7 \%$ of branches (Fig. 2b). In birds, BMR evolved at a

93 constant rate in $90.5 \%$ of branches and at faster rates in $9.5 \%$ of branches (Fig. $2 \mathrm{~d}$ ).

94 Avian $T_{\mathrm{b}}$ evolved at a constant rate in $69 \%$ of branches and at faster rates in $31 \%$ (Fig.

$952 \mathrm{e})$. When the branch-wise rates for $B M R$ and $T_{\mathrm{b}}$ were compared, we found that in

96 mammals, both traits evolved at a constant rate in $10.6 \%$ of branches (Fig. 3a consistent

97 with Fig. 1a). In $60.2 \%$ of branches only one trait evolved at faster rates while the other

98 trait diverged at a constant rate. This indicates that $B M R$ and $T_{\mathrm{b}}$ evolved in a decoupled

99 fashion along these branches (Fig. 3a consistent with Fig. 1d, e). We found that 29.2\% of

100 branches had an increased rate in both $B M R$ and $T_{\mathrm{b}}$. However, the magnitudes of the

101 branch-wise rates were not significantly correlated ( $p_{\mathrm{MCMC}}[\%$ of posterior distribution 
102 crossing zero] $=9 \%$; Table S9; Fig. 3a consistent with Fig 1f). This also suggests

103 decoupled evolution in those branches - likely because of distinct selection pressures

104 acting on $B M R$ and $T_{\mathrm{b}}$. On the other hand, both traits evolved at a constant rate in $63.8 \%$

105 of branches for birds (Fig. 3c consistent with Fig. 1a). In 32\% of branches only one trait

106 evolved at fast rates while the other trait diverged at a constant rate (Fig. 3c consistent

107 with Fig. 1d, e). In the remaining $4.2 \%$ of branches, both traits evolved at faster rates, but

108 the $r$ magnitudes were not statistically correlated $\left(p_{\mathrm{MCMC}}=16.9 \%\right.$, Table S10, Fig. 3c

109 consistent with Fig. 1f).

110

111 As rapid bursts in $B M R$ evolution were not coupled with those in $T_{\mathrm{b}}$ evolution, we

112 evaluated the alternative hypothesis postulating that $B M R$ evolved in response to $T_{\mathrm{a}}$. This

113 hypothesis suggests that colder environments increase the rate of heat lost from

114 organisms which is subsequently compensated by $B M R$ increases ${ }^{9-12}$. These $B M R$

115 increases could have occurred over long periods of time because of global cooling ${ }^{18}$ -

116 generating a long-term directional trend in BMR during the radiation of mammals and

117 birds. This expectation is in line with the Plesiomorphic-Apomorphic Endothermy

$118 \mathrm{Model}^{6-8}$ (PAE Model). By assuming that $B M R$ and $T_{\mathrm{b}}$ are coupled in endotherms and that

119 they both can be used as a proxy of the degree of endothermy, the PAE model predicts a

120 general tendency towards higher endothermic levels through time (from basoendothermic

121 ancestors, Methods) associated with the Cenozoic global cooling. Global cooling is not

122 the only source of variation in $T_{\mathrm{a}}$. Long-term directional increases in $B M R$ might have

123 also been driven by historical dispersals of endotherms towards higher latitudes ${ }^{19}$. In

124 either case, if a long-term decrease in $T_{\mathrm{a}}$ drove adaptation via $B M R$ elevation, and $T_{\mathrm{b}}$ 
125 followed the same trajectory (as assumed by the PAE model) we expect to find a positive

126 correlation between the branch-wise rates of BMR and the branch-wise rates of $T_{\mathrm{a}}$. With

127 this in mind, we also expect a positive trend towards higher $B M R$ and $T_{\mathrm{b}}$ values from

128 basoendothermic ancestors and a negative trend towards lower $T_{\mathrm{a}}$ from warmer ancestral

129 environments. We used the variable-rates model to estimate the branch-wise rates for $T_{\mathrm{a}}$

130 whilst accounting for latitude since, generally, $T_{\mathrm{a}}$ decreases from the equator to the poles

131 (Methods; Table S11).

132

133 The variable-rates model significantly improved the fit to the $T_{\mathrm{a}}$ data over the constant-

134 rate regression model in both mammals and birds (Table S11). In 21.2\% of mammalian

135 branches $T_{\mathrm{a}}$ evolved at a constant rate, and with rate heterogeneity in the remaining

$13678.8 \%$ - including $72.2 \%$ of branches with faster rates and $6.6 \%$ with slower rates $(r<1$,

137 Fig. 2c). This indicates that most ancestral mammalian lineages (72.2\%) faced abrupt

138 historical changes in their $T_{\mathrm{a}}$, while far fewer lineages (6.6\%, mostly bats) survived and

139 continued existing in similar thermal environments. In birds, $77.6 \%$ of branches show

140 faster rates of $T_{\mathrm{a}}$ change, $22.1 \%$ show changes at a constant rate, and in only a single

141 branch the $T_{\mathrm{a}}$ changed at a slower rate (Fig. 2f).

143 When branch-wise rates of mammalian $B M R$ and $T_{\mathrm{a}}$ evolution were compared, we found

144 that they were coupled in $74.9 \%$ of branches $\left(p_{\mathrm{MCMC}}=0 \%\right.$; Table S12; Fig. 3b, consistent

145 with Fig. 1b). To evaluate further if $T_{\mathrm{a}}$ decreases were linked to $B M R$ increases in the

$14674.9 \%$ of mammals where both traits were coupled (i.e. to ascertain the direction of

147 change), we evaluated the expected positive trend in $B M R$ as a response to the long-term 
148 decrease in $T_{\mathrm{a}}$. We conducted Bayesian phylogenetic regressions between extant values

149 of these two variables (in turn) and the path-wise rates (sum of branch-wise rates along

150 branches in the path from the root of the tree to each terminal species, Methods $)^{15}$. We

151 found a negative effect of path-wise rates on $T_{\mathrm{a}}$ across all mammals (Fig. 4b; Table S14),

152 which supports a long-term directional trend towards habitats with lower $T_{\mathrm{a}}$ over time.

153 However, we did not find evidence for any trend in mammalian $B M R$ evolution $-B M R$

154 increases and decreases were equally likely in our sample (Table S14). Our results

155 suggest that in colder environments, where resources were available to fuel metabolic

156 elevation, selection favoured higher mammalian $B M R^{20}$. Another possibility might be that

$157 B M R$ increase was a correlated response to direct selection on other physiological traits,

158 like maximal metabolic capacities for thermogenesis, whose benefits outweigh the

159 energetic cost of $B M R$ elevation ${ }^{20}$. Otherwise, selection may have always favoured $B M R$

160 decreases under an ever colder environment ${ }^{20}$.

161

162 In contrast to mammals, most avian branches that experienced rapid shifts in $T_{\mathrm{a}}$ did not

163 show evidence of coupled changes in $B M R-68.4 \%$ of branches had fast rates of $T_{\mathrm{a}}$

164 evolution but a constant rate of $B M R$ evolution (Fig. 3d consistent with Fig. 1d, e).

165 Moreover, the small fraction of branches where BMR evolved at fast rates $(9.5 \%)$ were

166 not linked to rapid shifts in $T_{\mathrm{a}}$ (Fig. 3d consistent with Fig. 1f; Table S13). Avian BMR

167 did not show a positive evolutionary trend despite the fact they also experienced colder

168 environments over time (Fig. 4d; Table S15). Birds might not have responded to colder

169 temperatures by changes in their $B M R$ because their lower thermal conductance might 
170 have helped them retain internal heat ${ }^{9}$. Alternatively, other physiological strategies, such

171 as torpor, may have been selected for under colder environments ${ }^{21}$.

172

173 Finally, we found a negative effect of path-wise rates on $T_{\mathrm{b}}$ in both mammals (Fig. 4a;

174 Table S14) and birds (Fig. 4c; Table S15). This suggest that - on average - endotherms

175 evolved towards colder bodies from warmer-bodied ancestors. These directional models

176 predict a mean $T_{\mathrm{b}}$ of $35.3{ }^{\circ} \mathrm{C}$ and $40.4{ }^{\circ} \mathrm{C}$ in the most recent common ancestor (MRCA)

177 of mammals and birds respectively (Fig. 4a, c), suggesting that early birds and mammals

178 were mesoendotherm rather than basoendotherms (Methods). This result does not support

179 that ancestral mammals could not attain $T_{\mathrm{b}}>30{ }^{\circ} \mathrm{C}$ owing to the elevated metabolic rates

180 necessary to compensate heat loss in cold environments ${ }^{22}$. However, if the $T_{\mathrm{b}}-T_{\mathrm{a}}$

181 differential $(\Delta T)$ determines how hot early mammals were, we expect that the mammalian

182 MRCA with a $T_{\mathrm{b}}$ of $35.3{ }^{\circ} \mathrm{C}$ could survive in an environment warm enough to have a low

$183 \Delta T$. Our model describing the negative trend in $T_{\mathrm{a}}$ predicts that the MRCA of mammals

184 lived in an environment with $23^{\circ} \mathrm{C}$ on average (Fig. 4b), resulting in a $\Delta T$ of $15.3^{\circ} \mathrm{C}$.

185 This ancestral $\Delta T$ is very conservative compared with the $\Delta T$ s observed in extant

186 mammals. For example, there are small mammals that achieve $T_{\mathrm{b}}$ higher than $39^{\circ} \mathrm{C}$ (e.g.

187 Microdipodops pallidus $\left.{ }^{16}\right)$ that can survive in environments of $11^{\circ} \mathrm{C}^{19}\left(\Delta T=28^{\circ} \mathrm{C}\right)$.

188 Also, some larger mammals have stable $T_{\mathrm{b}}$ even in extreme environmental conditions -

189 the Artic hare (Lepus arcticus) can maintain its $T_{\mathrm{b}}$ of $38^{\circ} \mathrm{C}^{16}$ in temperatures as low as -

$19012{ }^{\circ} \mathrm{C}^{19}\left(\Delta T=50^{\circ} \mathrm{C}\right)$. 
192 Taken together, our results reveal that $B M R$ was not coupled with $T_{\mathrm{b}}$ across the evolution

193 of endothermic species. As environments became colder, mammals survived by changing

194 their BMR, while birds likely survived owing to their high thermal insulation. Evaluating

195 the isolated and/or combined effect of environmental variables on physiological attributes

196 has implications for evidence-based projections for the future ${ }^{23}$. In this sense, the

197 previously unappreciated complexity, interplay and decoupled nature in the evolutionary

198 history of $B M R, T_{\mathrm{b}}$ and $T_{\mathrm{a}}$ might point to undetected resilience of endotherms in the face

199 of modern global challenges.

$201 \quad$ Figure legends.

202

203 Figure 1. Possible evolutionary scenarios between $\boldsymbol{B M R}$ and $\boldsymbol{T}_{\mathrm{b}}$ given their branch-

204 wise rates (r) in a bivariate space. Grey colours represent the constant background rate

$205(r=1)$. Red colours represent rates faster than the background rate $(r>1)$ and blue

206 colours represent rates slower than the background rate $(r<1)$, which might be related to

207 past events of positive ${ }^{17}$ and stabilizing selection ${ }^{29}$ respectively. Point fill colours

208 represent $B M R$ rates and point outline colours represent $T_{\mathrm{b}}$ rates.

210 Figure 2. Branch-wise rates $(\boldsymbol{r})$ of $B M R, T_{\mathrm{b}}$, and $T_{\mathrm{a}}$ on the mammalian and avian

211 phylogeny. Silhouettes courtesy of Phylopic/Apokryltaros (vectorized by T. Michael

212 Keesey), Becky Barnes, Doug Backlund et al., Emily Willoughby, Enoch Joseph et al.,

213 Estelle Bourdon, Ferran Sayol, FunkMonk, Jon Hill (Photo by DickDaniels), L. Shyamal, Lip

214 Kee Yap (modified), Mathew Callaghan, Matt Martyniuk, nicubunu, Pearson Scott 
215 Foresman, Prin Pattawaro et al., Rebecca Groom, Sarah Werning, T. Michael Keesey

216 (after Joseph Wolf), Yan Wong, Steven Traver. Silhouette licence links:

217 https://creativecommons.org/licenses/by/3.0/;

218 https://creativecommons.org/licenses/by-sa/3.0/.

220 Figure 3. Branch-wise rates $(r)$ of $B M R, T_{b}$, and $T_{\mathrm{a}}$ in bivariate space for mammals

221 (a, b) and birds (c, d). Bayesian GLS analyses indicates that only fast branch-wise rates

222 for $B M R$ and slow-fast branch-wise rates of $T_{\mathrm{a}}$ were statistically correlated in mammals

$223 \quad\left(p_{\mathrm{MCMC}}=0 ; \mathrm{n}=602\right.$ branches; black line $)$.

224

225 Figure 4. Mammals (a, b) and birds (c, d) evolved towards both colder $T_{\mathrm{b}}$ and $T_{\mathrm{a}}$

226 over their evolutionary history. Path-wise rates had a significant negative effect in

227 mammalian and avian $T_{\mathrm{b}}\left(p_{\mathrm{MCMC}}=4 \%\right.$ and $3 \% ; \mathrm{n}=502$ and 367 species $)$ and in

228 mammalian and avian $T_{\mathrm{a}}\left(p_{\mathrm{MCMC}}=0\right.$ and $0 ; \mathrm{n}=2922$ and 6142 species $)$, both supporting

229 a negative macroevolutionary trend ${ }^{15}$. Transparent and dark lines indicate the posterior

230 distribution of slopes and the mean slope respectively, estimated from the Bayesian

231 PGLS (Methods).

232

233 Methods.

234 Data. We used a time-calibrated phylogenetic tree of extant mammals $(n=3321)^{24}$, and

235 the body mass $(M)$, basal metabolic rate $(B M R)$, and body temperature $\left(T_{\mathrm{b}}\right)$ taken from

236 Clarke et al. ${ }^{16}(n=632)$. After identifying species in the tree that have trait information, 
237 we obtained a final mammalian dataset of 502 species, which includes representatives

238 from 15 orders (SI).

240 For birds, we used the consensus time-calibrated tree from Rolland et al. ${ }^{19}$. This tree was

241 inferred from the samples of trees provided by Jetz et $\mathrm{al}^{25}$. Data for $B M R, T_{\mathrm{b}}$, and Mass

242 were obtained from Fristoe et $\mathrm{al}^{9}$. After matching this database with the phylogenetic

243 tree, we obtained a final sample of 164 species which includes representatives from 21

244 orders (SI). The dataset used to evaluate evolutionary trends in $T_{\mathrm{b}}$ (see below) is from

245 Clarke \& Rothery ${ }^{26}$, which contains 367 species with phylogenetic information.

247 Data for ambient temperature $\left(T_{\mathrm{a}}\right)$ and latitude for extant mammals and birds was

248 extracted from Rolland et al. ${ }^{19}$ These datasets include 2922 species of mammals and 6142

249 species of birds which have phylogenetic information. The $T_{\mathrm{a}}$ for extant endothermic

250 species is the temperature of environments in which birds and mammals inhabit today -

251 measured as the mean ambient temperature for the mid-point latitude of each species

252 distribution (Rolland et al. ${ }^{19}$ ). The $T_{\mathrm{a}}$ at which a species exists today may not be a

253 heritable trait per se. However, the evolution of $T_{\mathrm{a}}$ can still be inferred using phylogenetic

254 methods since habitat selection reflects species adaptations (traits) to some characteristics

255 of the environment. This interrelationship should leave phylogenetic signal in the $T_{\mathrm{a}}$ at

256 which endothermic species live. Accordingly, we found significant phylogenetic signal in

257 the $T_{\mathrm{a}}$ of both mammals $\left(\lambda_{\text {PosteriorMean }}=0.77\right.$; Bayes Factor $\left.=665\right)$ and birds $\left(\lambda_{\text {PosteriorMean }}=\right.$

2580.8 ; Bayes Factor $=1404)$. Furthermore, the phylogenetic signal for $T_{\mathrm{a}}$ is very high $(\lambda=1)$

259 in birds and mammals, when estimated using the median- $r$ scaled tree. 
261 Finally, to evaluate the endothermic levels for the MRCA of mammals and birds

262 proposed by Lovegrove ${ }^{7,8}$, we followed his categorization of endothermic species as

263 basoendotherms $\left(T_{\mathrm{b}}{ }^{\text {Birds }}<40.4{ }^{\circ} \mathrm{C} ; T_{\mathrm{b}}{ }^{\text {Mammals }}<35.0^{\circ} \mathrm{C}\right)$, mesoendotherma $\left(40.4{ }^{\circ} \mathrm{C} \leq\right.$

$\left.264 T_{\mathrm{b}}{ }^{\text {Birds }} \leq 42.5^{\circ} \mathrm{C} ; 35^{\circ} \mathrm{C} \leq T_{\mathrm{b}}{ }^{\text {Mammals }} \leq 37.9^{\circ} \mathrm{C}\right)$, and supraendotherms $\left(T_{\mathrm{b}}{ }^{\text {Birds }}>42.5^{\circ} \mathrm{C}\right.$;

$\left.265 T_{\mathrm{b}}{ }^{\text {Mammals }}>37.9{ }^{\circ} \mathrm{C}\right)$.

267 Inferring the branch-wise rates of evolution. We identified heterogeneity in the rate of

268 evolution along phylogenetic branches (branch-wise rates) by dividing the rate into two

269 parameters: a background rate parameter $\left(\sigma_{b}^{2}\right)$ which assumes changes in the trait of

270 interest (e.g. BMR) are drawn from an underlying Brownian process, and a second

271 parameter, $r$, that identifies a branch-specific rate shift. A full set of branch-wise rates are

272 estimated by adjusting the lengths of each branch in a time-calibrated tree (stretching or

273 compressing a branch is equivalent to increasing or decreasing the phenotypic rate of

274 change relative to the underlying Brownian rate of evolution). Branch-wise rates are

275 defined by a set of branch-specific scalars $r(0<r<\infty)$ which transform each branch in

276 order to optimize the phenotypic rate of change to a Brownian process $\left(\sigma_{\mathrm{b}}^{2} r\right)$. If

277 phenotypic change occurred at accelerated (faster) rates along a specific branch of the

278 tree, then $r>1$ and the branch is stretched. Decelerated (slower) rates of evolution are

279 detected by $r<1$ and the branch is compressed. If the trait evolves at a constant rate

280 along a branch, then the branch will not be modified (i.e. $r=1)$. 
282 We estimated the $r$ values of $B M R, T_{\mathrm{b}}$, and $T_{\mathrm{a}}$ evolution using the phylogenetic variable-

283 rates regression model in a Bayesian framework ${ }^{17}$. This model is designed to

284 automatically detect shifts in the rate of trait evolution across phylogenetic branches

285 while accounting for a relationship with another trait or traits across extant species

286 values. This approach allows for simultaneous estimation of both an overall relationship

287 between, for instance, $B M R$ as a function of Mass and $T_{\mathrm{b}}$ across extant species, and any

288 shifts in rates ( $r$ ) that apply to the phylogenetically structured residual variance in the

289 relationship. As residual variance is explained by shifts in rate across phylogenetic

290 branches we can, for example, determine how much BMR has changed in the past $(r)$

291 after accounting for their covariation with Mass and $T_{\mathrm{b}}$ in the present (the relationship

292 between the values across extant species). Thus, if the amount of $B M R$ change along

293 individual phylogenetic branches were coupled with the amount of change of $T_{\mathrm{b}}$, then we

294 should find the $r$ values of $B M R$ to be positively associated with the $r$ values of $T_{\mathrm{b}}$. The

295 branch-wise rates for $T_{\mathrm{b}}$ evolution can be estimated while accounting for its covariation

296 with other traits or factor across extant species. Previous studies on the association

297 between $B M R$ and $T_{\mathrm{b}}$ using extant species values alone have not evaluated the association

298 in evolutionary terms even when they use phylogenetic method.

300 We evaluated 24 phylogenetic variable-rates regression models and 24 phylogenetic

301 constant-rate regression models (Table S1 to S8). Regression model selection was

302 conducted using Bayes Factors $(B F)$ via marginal likelihoods estimated by stepping stone

303 sampling. $B F$ is calculated as the double of the difference between the log marginal-

304 likelihood of the complex model and the simple model. By convention, $B F>2$ indicates 
305 positive evidence for the complex model, $B F$ 5-10 indicates strong support, and $B F>10$

306 are considered very strong support ${ }^{27}$. We inferred the $r$ values of $B M R$ and $T_{\mathrm{b}}$ with the

307 phylogenetic variable-rates regression models that best fit the data for our samples of

308 mammals and birds (Table S7 and S8). We also estimated the $r$ values for $T_{\mathrm{a}}$ after

309 accounting for the effect of latitude of species distribution (Table S11) and, consequently,

310 we accounted for the geographic variation of $T_{\mathrm{a}}$ across extant species distributions. We

311 used BayesTraits v3.028 to detect the magnitude and location of $r$ in a Bayesian Markov

312 chain Monte Carlo (MCMC) reversible-jump framework, which generates a posterior

313 distribution of trees with scaled branches lengths according to the rate of evolution. There

314 is no limit or prior expectation in the number of the $r$ branch-scalars, $r$ numbers vary

315 from zero (no branch is scaled) to $n$, where $n$ is the number of branches in the

316 phylogenetic tree. Regarding the values of each $r$ parameter, we used a gamma prior,

317 with $\alpha=1.1$ and $\beta$ parameter rescaled in order to get the median of the distribution equal

318 to one. With this setting, the numbers of rate increases and decreases proposed is

319 balanced $^{13}$. We ran 50,000,000 iterations sampling every 25,000 to ensure chain

320 convergence and independence in model parameters in $B M R$ and $T_{\mathrm{b}}$ analyses. We

321 discarded the first 25,000 iterations as burn in. For the $T_{\mathrm{a}}$ analysis in mammals we ran

$322200,000,000$ iterations sampling every 100,000, and we discarded the first 100,000

323 iterations as burn in. For $T_{\mathrm{a}}$ analysis in birds we ran 400,000,000 iterations discarding the

324 first 100,000,000 as burn in, and we sampled every 200,000. Regression coefficients

325 were judged as significant according to a calculated $p_{\mathrm{MCMC}}$ value for each posterior of

326 regression coefficients: where $<5 \%$ of samples in the posterior distribution crossed zero,

327 this indicates that the coefficient is significantly different from zero. 
329 Testing the relationship between the branch-wise rates of evolution. We first

330 estimated the consensus branch-scaled tree for $B M R$ and $T_{\mathrm{b}}$ from the posterior sample of

331 branch-scaled trees obtained with the phylogenetic variable-rates regression model. The

332 consensus branch-scaled tree was generated by using the median $r$ from the posterior

333 distribution. We evaluated the correlation between the $r$ values for $B M R$ and $T_{\mathrm{b}}$ using a

334 Bayesian GLS regression in BayesTraits v3.0. The same analyses were conducted to

335 evaluate the correlation between branch-wise rates for $B M R$ and $T_{\mathrm{a}}$. We used a uniform

336 prior for the $\beta$ (slope coefficient) ranging from -100 to 100 . We ran $50,000,000$ iterations

337 sampling every 25,000 to ensure chain convergence and independence in model

338 parameters. Significance of regression coefficients were determined as above.

340 Detecting trends. We evaluated the direction of change in $B M R, T_{b}$, and $T_{\mathrm{a}}$ across all

341 mammals and birds using the path-wise rates of these variables (Table S15 and S16).

342 Path-wise rate is the sum of all the branch-wise rates along the path of a species, which

343 lead from the root (the MRCA) to the tips of the tree, and it accounts for the total changes

344 the species has experienced during its evolution ${ }^{15}$. If high path-wise rates have

345 disproportionately been associated with trait increases or decreases, we expect to find that

346 species with greater path-wise rates will have high or low trait values in the present. For

347 instance, if ancestral mammals experienced progressively colder environmental

348 temperatures owing to climate change or colonization of colder habitats as they were

349 evolving from their MRCA, we expect a negative correlation between the path-wise rate

350 of $T_{\mathrm{a}}$ and the $T_{\mathrm{a}}$ of extant species. We performed six Bayesian PGLS regressions in 
351 BayesTraits 3.0 to evaluate the relationship between $B M R, T_{b}, T_{\mathrm{a}}$ and their path-wise

352 rates (Table S15 and S16). We used a uniform prior for the $\beta$ (slope coefficients) ranging

353 from -100 to 100 to allow all possible values to be equally likely. Finally, we ran

$35450,000,000$ iterations sampling every 25,000 to ensure chain convergence and

355 independence in model parameters. Significance of regression slopes were determined as

356 above.

357

358 End notes.

359 Supplementary Information is linked to the online version of the paper at

360 www.nature.com/nature.

361

362 Acknowledgments: We thank Ciara O’Donovan, Joanna Baker, Manabu Sakamoto and

363 Ana N. Campoy for helpful discussion on the manuscript. We also thank to three

364 anonymous reviewers who made valuable contributions. Andrew Clarke kindly supplied

365 data for mammals and birds. This work was part of the J.A-LL. PhD thesis, supported by

366 the CONICYT Doctoral Fellowships \#21130943. CV was supported by the Leverhulme

367 Trust (RPG-2013-185 and RPG-2017-071). C.E.U and E.R.S were supported by

368 FONDECYT grants \#1170815 and \#1170486.

369

370 Authors contribution. J.A-LL., C.E.U., E.R.S, and C.V., contributed to all aspect of this 371 work.

372

373 Author information: We have no competing interests. 
375 Data availability statement. Correspondence and request for materials should be

376 addressed to J.A-LL. (jorgeavariall@gmail.com) or C.V. (c.d.venditti@reading.ac.uk).

377

$378 \quad$ References

379

380 1. Clarke, A. Principles of Thermal Ecology. Temperature, Energy and Life. (Oxford 381 University Press, 2017)

382

383 2. Gillooly, J. F., Brown, J. H., West, G. B., Savage, V. M. \& Charnov, E. L. Effects of 384 size and temperature on metabolic rate. Science 293, 2248-2251 (2002).

3. Clarke, A., \& Pörtner H-O. Temperature, metabolic power and the evolution of

387 endothermy. Biol. Rev. 55, 703-727 (2010).

389 4. Kemp, T. S. The origin of mammalian endothermy: a paradigm for the evolution of 390 complex biological structure. Zool. J. L. Soc. 147, 473-488 (2006).

392 5. Brown, J. H., Gillooly, J. F., Allen, A. P., Savage, V. M. \& West, G. B. Towards a 393 metabolic theory of ecology. Ecology 85, 1771-1789 (2004).

395 6. Lovegrove, B. G. The evolution of endothermy in Cenozoic mammals: a 396 plesiomorphic-apomorphic continuum. Biol. Rev. 87, 128-162 (2012). 
398 7. Lovegrove, B. G. The evolution of mammalian body temperature: the Cenozoic 399 supraendothermic pulses. J. Comp. Physiol. B. 182, 579-589 (2012).

400

401 8. Lovegrove, B. G. A phenology of the evolution of endothermy in birds and mammals.

402 Biol. Rev. 92, 1213-1240 (2017).

403

404 9. Fristoe, T. S. et al. Metabolic heat production and thermal conductance are mass405 independent adaptations to thermal environment in birds and mammals. PNAS 112. 406 15934-15939 (2015).

407

408 10. Naya, D. E., Naya, H., White, R. C. On the interplay among ambient temperature, 409 basal metabolic rate, and body mass. Am. Nat. 192, 518-524 (2018).

410

411 11. White, C. R., Blackburn, T. M., Martin G. R., Butler, P. J. Basal metabolic rate of 412 birds is associated with habitat temperature and precipitation, not primary productivity. 413 Proc. Biol. Sci. 274, 287-293 (2007).

414

415 12. Jetz, W., Freckleton, R. P., McKechnie, A. E. Environment, migratory tendency, 416 phylogeny and basal metabolic rate in birds. PLoS ONE 3(9):e3261. 417 doi:10.1371/journal.pone.0003261 (2008). 
419 13. Venditti, C., Meade, A. \& Pagel, M. Multiples routes to mammalian diversity. Nature 420 479, 393-396 (2011).

421

422 14. Rabosky, D. L. Automatic detection of key innovations, rate shifts, and diversity423 dependence on phylogenetic trees. PLoS ONE 9:e89543 (2014).

425 15. Baker, J., Meade, A., Pagel, M. \& Venditti, C. Adaptive evolution toward larger size 426 in mammals. PNAS. 112, 5093-5098 (2015).

428 16. Clarke, A., Rothery, P. \& Isaac, N. J. B. Scaling of basal metabolic rate with body 429 mass and temperature in mammals. J. Anim. Ecol. 79, 610-619 (2010).

431 17. Baker, J., Meade, A., Pagel, M. \& Venditti, C. Positive phenotypic selection inferred 432 from phylogenies. Zool. J. Linn. Soc. 118, 95-115 (2016).

434 18. Zachos, J., Pagani, M., Sloan, L., Thomas, E. \& Billups, K. Trends, rhythms, and 435 aberrations in global climate 65 Ma to present. Science. 292, 686-693 (2001).

437 19. Rolland, J. et al. The impact of endothermy on the climatic niche evolution and the 438 distribution of vertebrate diversity. Nat. Ecol. Evol. 2, 459-464 (2018). 
440 20. Swanson, D. L., McKechnie, A. E., Vézina, F. How low can you go? An adaptive 441 energetic framework for interpreting basal metabolic rate variation in endotherms. J. 442 Comp. Physiol. B. 187, 1039-1056 (2017).

443

444 21. Körtner, G., Brigham, R. M. \& Geiser, F. Winter torpor in a large bird. Nature. 407, $445318(2000)$.

447 22. Crompton, A. W., Taylor, C. R. \& Jagger, J. A. Evolution of homeothermy in 448 mammals. Nature 272, 333-336 (1978).

450 23. Bozinovic, F. \& Pörtner, H-O. Physiological ecology meets climate change. Ecol. 451 Evol. 5, 1025-1030 (2015).

453 24. Fritz, S. A., Bininda-Emonds, O. R. \& Purvis, A. Geographical variation in predictors 454 of mammalian extinction risk: big is bad, but only in the tropics. Ecol Lett 12, 538-549 455 (2009).

456

457 25. Jetz, W., Thomas, G. H., Joy, J. B., Hartmann, K. \& Mooers, A. O. The global 458 diversity of birds in space and time. Nature. 491, 444-448.

460 26. Clarke, A. \& Rothery, P. Scaling of body temperature in mammals and birds. Func. 461 Ecol. 22, 58-67 (2008). 
463 27. Raftery, A.E. in Markov Chain Monte Carlo in Practice (eds Gilks, W. R., 464 Richardson, S. \& Spiegelhalter, D. J.) 163-187 (Chapman \& Hall, 1996).

465

466 28. Pagel, M., Meade, A. \& Barker, D. Bayesian estimation of ancestral character states 467 on phylogenies. Systematic Biology 53, 673-684 (2004).

468

469 29. Lieberman, B. S. \& Dudgeon, S. An evaluation of stabilizing selection as a 470 mechanism for stasis. Palaeogeography, Palaeoclimatology, Palaeoecology 127, 229-238 471 (1996). 